\title{
Padrões de prática de atividade física em adolescentes de um município da região Nordeste do Brasil
}

\author{
Standards of physical activity practices in adolescents \\ in a city of Northeastern Brazil
}

Gerfeson Mendonça ${ }^{1}$

Luanna Alexandra Cheng ${ }^{2}$

José Cazuza de Farias Júnior ${ }^{3}$

\footnotetext{
${ }^{1}$ Programa Associado de Pós-Graduação em Educação Física, Universidade Federal da Paraíba. Campus I, Cidade Universitária. 58059-900 João Pessoa PB Brasil. gerfeson_edf@hotmail.com ${ }^{2}$ Grupo de Estudos e Pesquisas em Epidemiologia da Atividade Física. João Pessoa PB Brasil.

${ }^{3}$ Universidade Federal da Paraíba. João Pessoa PB Brasil.
}

\begin{abstract}
The scope of this study was to describe the standards of physical activity practices and analyze its variations as sociodemographic characteristics of adolescents in a city of Northeastern Brazil. The sample consisted of 2,350 adolescents in the city of João Pessoa in the Brazilian State of Paraiba. The prevalence of physical activity was determined and the practice scores (minutes) week) calculated: general and types (sports, exercise, active commuting and recreational activities). Multiple linear regression was used to estimate the relative contribution of the types of practice for the general score of physical activity. The physical activities most practiced were active commuting (55.2\%) and sports (52.7\%). The activities that presented the greatest explanatory capacity for the overall score of practice were: sports (38.1\%) and exercises (21.8\%). The explanatory capacity of sports was increased in all subgroups, except in female patients. The practice of exercise contributes to the major changes in the general score in female adolescents from private schools. Recreational activities and active commuting had the largest contribution for the female gender. Sports and exercise were the activities that contributed the most to explain the changes in the general practice score.
\end{abstract}

Key words Adolescence, Motor activity, Socio-demographic factors, Brazil
Resumo O objetivo do presente estudo foi descrever os padrões de prática de atividade física e analisar suas variações conforme as características sociodemográficas de adolescentes de um município da região nordeste do Brasil. A amostra foi de 2.350 adolescentes do município de João Pessoa (PB). Foram determinadas as prevalências de atividade fisica e calculados os escores de prática (minutos/semana): geral e por tipos (esportes, exercícios físicos, deslocamento ativo e atividades recreativas). A regressão linear foi utilizada para estimar a contribuição relativa dos tipos de prática para o escore geral de atividade física. As atividades mais praticadas foram: deslocamentos $(55,2 \%)$ e esportes $(52,7 \%)$. As atividades que apresentaram maior capacidade explicativa para o escore geral de prática foram: esportes $(38,1 \%) e$ exercícios físicos (21,8\%). A capacidade explicativa dos esportes foi elevada em todos os subgrupos, exceto no sexo feminino. A prática de exercícios físicos contribuiu para as maiores variações no escore geral nos adolescentes do sexo feminino e de escolas privadas. As atividades recreativas e o deslocamento ativo tiveram a maior contribuição para o sexo feminino. Esportes e exercícios foram as atividades que mais contribuíram para explicar as variações no escore geral de prática.

Palavras-chave Adolescência, Atividade motora, Fatores sociodemográficos, Brasil 


\section{Introdução}

Nos dez últimos anos, foi publicado um grande volume de estudos sobre nível de atividade física em adolescentes ${ }^{1,2}$. Esses estudos demonstraram uma elevada prevalência de adolescentes que não atendiam às recomendações de prática de atividade física para a saúde $e^{1,2}$.

Identificar as prevalências de práticas de atividade física é importante para se conhecer o cenário sobre a participação das pessoas, seu comportamento temporal, identificar seus determinantes e analisar sua associação com desfechos em saúde ${ }^{2}$. No entanto, tais informações não permitem conhecer os padrões de prática de atividade física, como tipo, frequência, duração das atividades e a contribuição dos diferentes tipos de prática para o escore geral de atividade física, bem como suas variações em função das características sociodemográficas dos adolescentes.

Os estudos sobre padrões de atividade física em adolescentes têm frequentemente investigado o tipo ${ }^{3,4}$, a frequência ${ }^{3-5}$ e a duração $0^{4}$ das atividades mais praticadas e suas contribuições para o escore geral ${ }^{4}$. Revisões sistemáticas ${ }^{1,2,6}$ demostraram que o nível geral de atividade física ou em determinados domínios (lazer, deslocamento e escola) varia com as características sociodemográficas dos adolescentes (sexo, faixa etária e condição socioeconômica). Porém, pouco se sabe se os padrões de atividade física dos adolescentes variam com essas características ${ }^{7,8}$.

Estudo de tendência temporal conduzido na região sul do Brasil demonstrou que as preferências e as atividades físicas mais praticadas pelos adolescentes sofreram mudanças no período de sete anos, caracterizadas pelo aumento na participação em exercícios físicos e pela redução da prática de esportes, principalmente em adolescentes do sexo feminino ${ }^{8}$. No entanto, as diferenças sociais, econômicas, culturais, climáticas e de estrutura física para a prática de atividade física entre as regiões do Brasil tornam mais difíceis a generalização desses achados para o país, em especial para a região Nordeste.

Identificar as atividades físicas mais realizadas pelos adolescentes, suas contribuições para o nível global/geral de atividade física e suas possíveis variações com as características sociodemográficas podem esclarecer os padrões de prática nesse grupo populacional. Essas informações são importantes para o desenvolvimento de programas de promoção da atividade física para adolescentes, principalmente no direcionamento de ações específicas que forneçam maior possibili- dade de práticas conforme o padrão de atividade física de cada subgrupo. Sendo assim, o objetivo do presente estudo foi descrever os padrões de prática de atividade física e analisar suas variações conforme características sociodemográficas de adolescentes de um município da região nordeste do Brasil.

\section{Métodos}

Este estudo foi baseado na análise dos dados de um estudo epidemiológico transversal, de base escolar, desenvolvido com amostra representativa de escolares do ensino médio, de ambos os sexos, de 14 a 19 anos de idade, de escolas públicas e privadas do município de João Pessoa (PB), região nordeste do Brasil, em 2009. O estudo foi aprovado pelo Comitê de Ética em Pesquisa com Seres Humanos da Universidade Federal da Paraíba e seguiu todos os procedimentos da Resolução no 196/96 do Conselho Nacional de Saúde.

Para o cálculo de tamanho da amostra considerou-se a prevalência de atividade física de 50\% (300 minutos ou mais por semana de prática de atividade física), intervalo de confiança de 95\%, erro máximo aceitável de três pontos percentuais, efeito de delineamento (deff) igual a dois e acréscimo de 30\% para compensar perdas e recusas. Com base nesses parâmetros, o tamanho da amostra foi estimado em 2.686 adolescentes.

A amostra foi selecionada por conglomerados em dois estágios: seleção sistemática de 30 escolas distribuídas proporcionalmente por tipo (públicas e privadas), tamanho (número de alunos) e região geográfica do município (norte, sul, leste, oeste); seleção aleatória de turmas para compor a amostra $(n=135)$, distribuídas proporcionalmente por turno (diurno e noturno) e série de ensino ( $1^{\mathrm{a}}, 2^{\mathrm{a}}$ e $3^{\mathrm{a}}$ séries do ensino médio).

A coleta de dados ocorreu entre maio e setembro de 2009. Os adolescentes responderam um questionário em sala de aula, no horário letivo regular, sendo orientados pela equipe de coleta de dados (previamente treinada e que seguiu um protocolo uniforme).

As variáveis sociodemográficas sexo (masculino e feminino), faixa etária (14-15 e 16-19 anos de idade), classe econômica (classe $\mathrm{A} / \mathrm{B}$ [alta] e classe $\mathrm{C} / \mathrm{D} / \mathrm{E}$ [média a baixa]) e tipo de escola (pública e privada) foram utilizadas para a caracterização da amostra e a estratificação das análises. Para determinar a classe econômica utilizou-se a metodologia da Associação Brasileira de Empresas de Pesquisa - ABEP', que considera 
a quantidade de bens materiais, presença de empregados mensalista no domicílio e a escolaridade do chefe da família.

A atividade física foi mensurada por questionário previamente validado ${ }^{10}$ e que era composto por uma lista de 24 atividades físicas (com possibilidade de acrescentar mais duas) de intensidade moderada a vigorosa e o deslocamento ativo (caminhada ou bicicleta). Os adolescentes referiram à frequência (dias/semana) e à duração (minutos/dia) de cada atividade física praticada por ao menos 10 minutos por sessão continua de prática. Para responder a essas questões, os adolescentes consideraram as atividades praticadas na semana anterior à coleta de dados. Foram calculados os seguintes escores de atividade física: escore geral de prática - determinado a partir do somatório dos produtos dos tempos despendido nas atividades pelas respectivas frequências de prática, resultando em um escore em minutos por semana; escores por cada um dos tipos de prática (minutos/semana): esportes (18 modalidades esportivas), exercícios físicos (5 tipos de exercícios), deslocamentos ativos (utilização de bicicleta ou caminhada como meio de deslocamento) e atividades recreativas ( 8 brincadeiras ao "ar livre"). Para o presente estudo será entendido como padrão de prática a descrição individual de cada atividade física, os tipos de prática (esportes, exercícios, deslocamentos e atividades recreativas), a participação semanal (\% frequência), a duração (média e mediana de minutos por semana), a contribuição relativa destas atividades para o escore geral de prática (minutos por semana) e as variações dessas conforme as características sociodemográficas dos adolescentes. Desta forma, as análises foram realizadas apenas com os adolescentes que praticaram pelo menos 10 minutos por semana.

As estatísticas descritivas incluíram os cálculos de prevalências e intervalos de confiança de 95\% (IC95\%) para as variáveis qualitativas e média, mediana e desvio padrão para as quantitativas. O teste do Qui-quadrado para heterogeneidade foi empregado para comparar a proporção de adolescentes que praticavam 10 minutos ou mais por semana de atividade física para o escore geral e por tipos de prática entre adolescentes de diferentes características sociodemográficas: sexo, faixa etária, classe econômica e tipo de escola.

A regressão linear múltipla foi utilizada para determinar a contribuição relativa dos diferentes tipos de prática (variáveis independentes) para o escore geral de atividade física (variável dependente), considerando as características sociode- mográficas dos adolescentes (sexo, faixa etária, classe econômica e tipo de escola). A correlação semiparcial foi utilizada para avaliar a contribuição única (percentual de variância explicada) de cada tipo de prática, bem como a "variância compartilhada" dessas práticas em relação ao escore geral de atividade física. Adolescentes que declararam realizar mais de 3.000 minutos de atividade física por semana ( $>7$ horas/dia) foram excluídos das análises (45 adolescentes).

As análises foram estratificadas por sexo, faixa etária, classe econômica e tipo de escola. Todas as análises foram realizadas no Stata 11.0 e consideraram o processo de seleção da amostra (opção svy). O nível de significância adotado foi de menos de $5 \%$ para testes bicaudais.

\section{Resultados}

A amostra final deste estudo foi composta por 2.350 adolescentes (recusas [2,0\%], perdas $[5,4 \%]$, exclusões $[11,2 \%]$ e adolescentes que não praticaram pelo menos 10 minutos por semana [13,8\%] totalizaram $32,4 \%$ dos casos), com idade média de 16,4 anos ( $\mathrm{DP}=1,2$ anos de idade). Não foram identificadas diferenças significativas nas características sociodemográficas, nível geral de atividade física e por tipos de prática entre os adolescentes que foram incluídos e excluídos das análises. A análise de poder estatístico a posteriori identificou que essa amostra permite estimar tamanho de efeito de 0,005 a 0,173, considerando até quatro preditores no modelo, nível de confiança de $95 \%$, beta $(\beta)$ de 0,05 e poder de $80,0 \%$.

A maioria dos adolescentes era do sexo feminino (55,2 \%), tinha entre 16 e 19 anos de idade $(69,0 \%)$, pertencia às classes econômicas de média a baixa (classe C/D/E: 54,1\%) e estudava em escolas públicas $(70,7 \%)$. A frequência de prática por tipos de atividades físicas (praticava 10 ou mais minutos por semana) é apresentada no Quadro 1. Entre os adolescentes que não praticaram nenhuma atividade física por pelo menos 10 minutos por semana (16,7\%; IC95\%: 15,3-18,0; $\mathrm{n}=479)$, a maioria era do sexo feminino $(74,5 \%)$ e de 16-19 anos de idade (68,7\%) - Dados não apresentados em tabela.

De forma geral, cerca de oito em cada dez adolescentes (83,3\%; IC95: 82,0-84,7) praticavam 10 minutos ou mais por semana de atividade física, sendo maior no sexo masculino e nos adolescentes mais jovens (14-15 anos de idade). O deslocamento ativo foi o tipo de atividade com a maior frequência de prática (55,2\%; IC95\%: 
53,1-57,2), particularmente no sexo feminino, na classe econômica mais baixa (classe $\mathrm{C} / \mathrm{D} / \mathrm{E}$ ) e nos de escolas públicas. Os esportes tiveram a segunda maior participação (52,7\%; IC95\%: 50,7-54,7), com destaque para os adolescentes do sexo masculino, os mais jovens e os de classe econômica mais alta (classe A/B). A terceira maior frequência de prática foi para os exercícios físicos (50,5\%; IC95\%: 48,5-52,5), sobretudo nos adolescentes do sexo masculino, de classe econômica mais alta e que estudavam em escolas privadas. As atividades recreativas foram as menos praticadas pelos adolescentes (41,5\%; IC95\%:
39,5-43,5). Porém, os que estudavam em escolas públicas foram os que mais praticavam esse tipo de atividade (Tabela 1 ).

O tempo mediano de prática de atividade física dos adolescentes foi de 420 minutos por semana (média: 619,7; DP = 600,2). A mediana do tempo de participação em modalidade esportiva, exercícios físicos, deslocamento ativo e atividades recreativas foram, respectivamente, 250 (média: $410,5 ; \mathrm{DP}=425,4), 210(324,7 ; \mathrm{DP}=322,6), 180$ (média: 284,8; DP = 326,1) e 150 (média: 219,4; $\mathrm{DP}=234,7)$ minutos por semana - Dados não publicados em tabelas.

Quadro 1. Frequência de participação dos adolescentes nas atividades físicas (prática de 10 minutos ou mais por semana), município de João Pessoa (PB), 2009.

\begin{tabular}{|c|c|}
\hline $\begin{array}{l}\text { Tipos de práticas de } \\
\text { atividades físicas }\end{array}$ & Atividades e frequência de prática (\%) \\
\hline Esportes & $\begin{array}{l}\text { Futsal }(22,3 \%) \text {, futebol }(21,6 \%) \text {, voleibol }(16,5 \%) \text {, natação }(5,5 \%) \text {, futebol de areia } \\
(5,1 \%) \text {, basquetebol }(4,9 \%) \text {, vôlei de praia }(4,7 \%) \text {, praticas de lutas ou artes maciais } \\
(4,6 \%) \text {, handebol }(4,5 \%) \text {, atletismo }(3,1 \%) \text {, surfe }(2,3 \%) \text {, ginastica olímpica ou } \\
\text { rítmica }(1,1 \%) \text {, tênis }(0,8 \%) \text { e outros esportes }(0,7 \%)^{*} \text {. }\end{array}$ \\
\hline Exercícios físicos & $\begin{array}{l}\text { Exercícios calistênicos }(23,4 \%)^{* *} \text {, caminhar }(20,5 \%) \text {, correr }(19,3 \%) \text {, musculação } \\
(15,0 \%) \text { e ginástica em academia }(3,5 \%) \text {. }\end{array}$ \\
\hline Deslocamento ativo & $\begin{array}{l}\text { Caminhar ou usar bicicleta como meio de transporte para ir à escola, casa de amigos, } \\
\text { supermercados entre outros deslocamentos }(56,1 \%) \text {. }\end{array}$ \\
\hline Atividades recreativas & $\begin{array}{l}\text { Andar de bicicleta / pedalar }(22,2 \%) \text {, danças }(12,6 \%) \text {, jogar queimado / baleado / } \\
\text { pular corda }(4,3 \%) \text {, andar de patins / skate }(3,7 \%) \text { e outras atividades recreativas } \\
(0,3 \%)^{* * *} \text {. }\end{array}$ \\
\hline
\end{tabular}

* Tênis de mesa, futebol americano, hipismo, polo aquático e nado sincronizado; ${ }^{\star \star}$ Exercícios abdominais, flexões de braços e pernas; ${ }^{* *}$ Realizar trilha, Le Parku, jogar frescobol e barra-bandeira.

Tabela 1. Prevalência de prática de atividade física (prática de 10 minutos ou mais por semana) por tipos e características sociodemográficas dos adolescentes do município João Pessoa (PB), 2009.

\begin{tabular}{|c|c|c|c|c|c|c|c|c|c|c|c|c|c|c|c|}
\hline \multirow[t]{2}{*}{ Variáveis } & \multicolumn{3}{|c|}{$\begin{array}{l}\text { Escore global de } \\
\text { atividade física }\end{array}$} & \multicolumn{3}{|c|}{ Esportes } & \multicolumn{3}{|c|}{ Exercícios físicos } & \multicolumn{3}{|c|}{ Deslocamento ativo } & \multicolumn{3}{|c|}{$\begin{array}{l}\text { Atividades } \\
\text { recreativas }\end{array}$} \\
\hline & $\mathbf{n}$ & $\%$ & $p^{*}$ & $\mathbf{n}$ & $\%$ & $\mathbf{p}^{*}$ & $\mathbf{n}$ & $\%$ & $\mathbf{p}^{*}$ & $\mathbf{n}$ & $\%$ & $\mathrm{p}^{*}$ & $\mathrm{n}$ & $\%$ & $\mathbf{p}^{*}$ \\
\hline Todos & 2.350 & 83,1 & & 1.239 & 52,7 & & 1.187 & 50,5 & & 1.296 & 55,2 & & 975 & 41,5 & \\
\hline Sexo & & & $<0,001$ & & & $<0,001$ & & & $<0,001$ & & & $<0,001$ & & & 0,676 \\
\hline Masculino & 1.045 & 89,6 & & 792 & 75,7 & & 603 & 57,7 & & 476 & 45,5 & & 440 & 42,1 & \\
\hline Feminino & 1.291 & 78,3 & & 438 & 33,9 & & 580 & 44,9 & & 813 & 63,0 & & 532 & 41,2 & \\
\hline Faixa etária & & & $<0,001$ & & & 0,001 & & & 0,279 & & & 0,599 & & & 0,083 \\
\hline 14-15 anos & 955 & 86,4 & & 543 & 56,8 & & 470 & 49,2 & & 521 & 54,5 & & 417 & 43,6 & \\
\hline 16-19 anos & 1.394 & 80,9 & & 696 & 49,9 & & 717 & 51,4 & & 775 & 55,6 & & 558 & 40,0 & \\
\hline $\begin{array}{l}\text { Classe } \\
\text { econômica }\end{array}$ & & & 0,621 & & & 0,003 & & & 0,017 & & & 0,006 & & & 0,112 \\
\hline $\mathrm{A} / \mathrm{B}$ & 959 & 84,1 & & 537 & 56,0 & & 510 & 53,2 & & 511 & 53,3 & & 384 & 40,0 & \\
\hline $\mathrm{C} / \mathrm{D} / \mathrm{E}$ & 1.128 & 83,4 & & 559 & 49,5 & & 541 & 47,9 & & 669 & 59,3 & & 491 & 43,5 & \\
\hline Tipo de escola & & & 0,635 & & & 0,699 & & & 0,013 & & & 0,007 & & & 0,031 \\
\hline Privada & 688 & 82,5 & & 367 & 53,4 & & 375 & 54,5 & & 350 & 50,9 & & 262 & 38,1 & \\
\hline Pública & 1.661 & 83,3 & & 872 & 52,5 & & 812 & 48,9 & & 946 & 56,9 & & 713 & 42,9 & \\
\hline
\end{tabular}

${ }^{\star}$ Teste do Qui-quadrado para heterogeneidade. 
A participação em modalidades esportivas foi responsável pela maior variância no escore geral de atividade física $(38,1 \%)$ dos adolescentes. Os valores de contribuição relativa dos esportes para o escore geral de prática foram acima de 35\% em todos os subgrupos das variáveis sociodemográficas, exceto no sexo feminino $(18,2 \%)$. Os exercícios físicos foram as atividades com a segunda maior capacidade explicativa (21,8\%), particularmente no sexo feminino, nos que estudavam em escolas privadas, nos adolescentes mais velhos (16-19 anos de idade) e nos de classe econômica alta. As atividades recreativas tiveram a terceira maior capacidade explicativa $(17,6 \%)$, com maior contribuição relativa para os adolescentes do sexo feminino. $\mathrm{O}$ deslocamento ativo foi a atividade com menor capacidade explicativa $(11,9 \%)$, exceto no sexo feminino $(20,6 \%)-\mathrm{Fi}$ gura 1.

Os subgrupos que mais praticaram simultaneamente diferentes tipos de atividade física (variância compartilhada) foram: os mais jovens $(13,9 \%)$, os que estudavam em escolas públicas $(13,6 \%)$, os de classe econômica mais baixa $(12,2 \%)$ e os do sexo masculino $(8,8 \%)$ - Figura 1.

\section{Discussão}

Este estudo descreveu os padrões de prática de atividades físicas e analisou suas variações de acordo com as características sociodemográficas de adolescentes de um município da região nordeste do Brasil. O deslocamento ativo e os esportes foram os tipos de atividades físicas mais praticadas pelos adolescentes. A prática de esportes e de exercícios físicos representaram as atividades que mais contribuíram para as variações no nível de atividade física. Porém, a contribuição relativa dos diferentes tipos de prática variou com as características sociodemográficas dos adolescentes.

A participação em esportes tem sido identificada como uma importante forma de prática de atividade física no lazer em adolescentes de todo mundo ${ }^{4,8,11}$. No presente estudo, quase metade dos adolescentes referiram praticar alguma modalidade esportiva e também foi o tipo de atividade que mais contribuiu isoladamente para variações no total do tempo de prática de atividade física que os adolescentes despenderam por semana, aproximadamente um terço do total do tempo de prática. Dois aspectos podem explicar esses achados: o primeiro está relacionado à utilização do esporte como conteúdo nas aulas

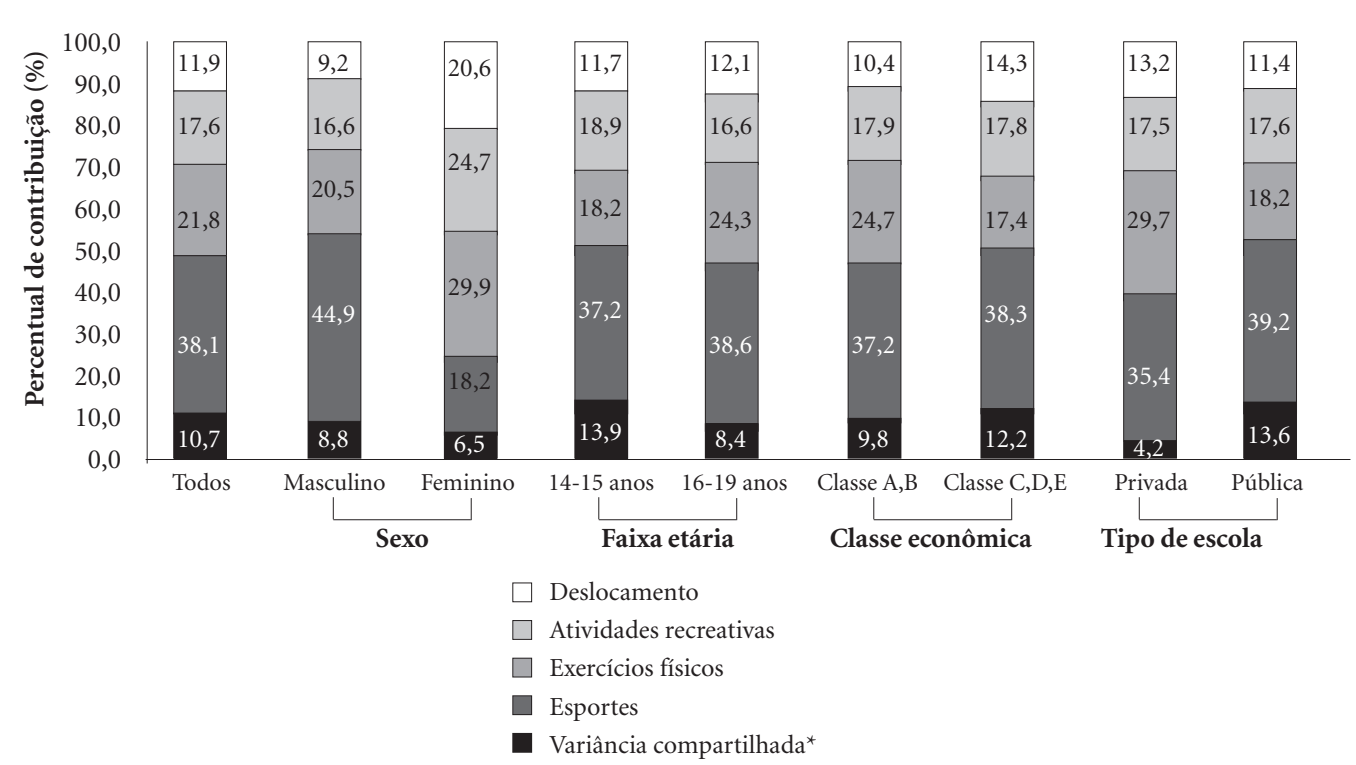

Figura 1. Contribuição explicativa dos tipos de práticas de atividades físicas para o escore global de atividade física (minutos/semana) de acordo com variáveis sociodemográficas em adolescentes do município de João Pessoa PB, 2009.

*Variância compartilhada $=$ escore de tempo que o adolescente tem de participação em mais de um tipo de atividade física. 
de educação física. De forma direta, o tempo de prática de esportes nessas aulas contribui para o tempo total de prática de atividade física (a própria participação do adolescente nas aulas). Indiretamente, as aulas de educação física estimularia a participação nessas modalidades fora do ambiente escolar (influenciada pelo aprendizado das aulas). Segundo, a prática de esportes é identificada como uma das atividades com maior preferência entre os adolescentes ${ }^{7,12}$ tendo em vista que são atividades desafiadoras, competitivas, praticadas de forma coletiva, na maioria das vezes $^{7,13}$, sendo mais atraentes para os adolescentes e que certamente contribuem para maior prática de ativada física destes.

O presente estudo identificou que cerca de cinco em cada dez adolescentes realizavam algum tipo de exercício físico durante a semana, com destaque para os de força $(38,4 \%$ calistênicos e musculação), caminhadas (20,5\%) e corridas $(19,3 \%)$. Esse tipo de atividade teve a segunda maior contribuição para o escore geral de atividade física, principalmente no sexo feminino $(29,9 \%)$ e em adolescentes de escolas privadas $(29,7 \%)$.

Ter identificado uma elevada contribuição do exercício físico para o escore geral de atividade física foi um importante achado do presente estudo. $\mathrm{O}$ aumento no interesse por atividades realizadas em academias (modalidades de ginástica aeróbica e a musculação), o envolvimento em corridas e caminhadas têm sido descrito em estudos de tendência temporal da prática de atividade física em adolescentes ${ }^{7,8}$. Em estudo desenvolvido com adolescentes do Sul do Brasil, Coll et al. ${ }^{8}$ não identificaram mudanças significativas na prevalência total de prática de atividade física no lazer entre os anos de 2005 e 2012. Entretanto, houve reduções entre 34\% e 58\% na participação dos adolescentes em modalidades esportivas e um aumento entre $55 \%$ e $128 \%$ na realização de exercícios físicos.

O aumento expressivo no interesse dos adolescentes por exercícios físicos pode ser decorrente do crescimento no número de academias de ginástica no país, o acesso das pessoas de média e baixa renda a essas academias, a falta ou a redução dos espaços públicos adequados para prática, a insegurança, o maior conhecimento sobre os benefícios da atividade física para a saúde, a aparente preocupação com o corpo (estética) e a disseminação e a valorização desse tipo de prática nos meios de comunicação ${ }^{7}$. É importante destacar também a menor participação dos adolescentes do sexo feminino em atividades esportivas dentro e fora do ambiente escolar ${ }^{8} \mathrm{e}$, em contrapartida, o aumento da participação em exercícios físicos ${ }^{8}$. Apesar da realização de exercícios físicos ter como um dos principais objetivos o desenvolvimento e/ou a manutenção dos níveis de aptidão física ${ }^{14,15}$. Na população adolescente, é possível também que a realização dessas atividades esteja associada à busca por um "corpo ideal", frequentemente enfatizado na sociedade contemporânea e na mídia ${ }^{16}$.

No presente estudo, as atividades recreativas foram as que apresentaram a menor proporção de praticantes, mas foi a responsável pela terceira maior capacidade explicativa do escore geral de atividade física, superando o deslocamento ativo. Este resultado demostra que estas atividades podem desempenhar um papel importante para aumentar o nível de atividade física dos adolescentes, tendo em vista que a contribuição relativa dessa prática se mostrou maior que o deslocamento ativo.

Em termos de capacidade explicativa nos subgrupos analisados, verificou-se que as atividades recreativas tiveram maior contribuição para o escore geral de atividade física no sexo feminino. Esse resultado pode estar relacionado ao fato de que estas adolescentes prefiram praticar atividades que não exijam equipamentos e locais específicos, regras fixas, habilidades específicas e as técnicas exigidas nos esportes. É importante considerar também que essas atividades são frequentemente identificadas como práticas advindas de forma espontânea, sem regras preestabelecidas, que não requerem espaços físicos e materiais específicos, e que têm como principal característica a diversão e o $\operatorname{prazer}^{17,18}$.

O deslocamento ativo é caracterizado como um importante domínio da prática de atividade física $^{1}$ e tem se associado positivamente a vários desfechos de saúde ${ }^{15,19}$. Embora mais da metade dos adolescentes do presente estudo realizassem algum tipo de deslocamento ativo $(55,1 \%)$, a capacidade explicativa deste tipo de atividade para o escore geral de atividade física foi baixa $(11,9 \%)$, comparada às demais. A explicação para esse resultado é que, embora muitos adolescentes usassem o deslocamento ativo em seus trajetos diários, na maioria das vezes era por um período de tempo muito curto $(84,4 \%$ dos adolescentes faziam menos de 210 minutos por semana [cerca de 30 minutos diários] - dados não publicados em tabelas), não influenciando significativamente no escore geral de atividade física.

A distância entre os locais parece ser um fator decisivo para o uso do deslocamento ativo entre 
os adolescentes. Estudos de revisões têm demostrado que a frequência de deslocamento ativo diminui conforme as distâncias entre a residência e a escola dos adolescentes são maiores ${ }^{20,21}$. Já em estudo desenvolvido com adolescentes canadenses Larouche et al..$^{22}$ identificaram que aqueles que residem muito próximo da escola sentem-se mais estimulados para ir a pé ou de bicicleta. No entanto, é importante considerar que embora as distâncias entre esses percursos estimulem a participação dos adolescentes nessas atividades, na prática elas são realizadas por um curto espaço de tempo, resultando em uma pequena contribuição para o tempo total de prática de atividade física. Além disso, é importante ressaltar que condições adequadas de infraestrutura e segurança no bairro permitem que o deslocamento ativo seja realizado por maiores distâncias pelos adolescentes. Estudos sobre ambiente e deslocamento ativo têm identificado que quanto melhor a estrutura de ruas, calçadas, conectividade das ruas e a segurança nos bairros, mais comumente os adolescentes se deslocam de forma ativa para ir e viri, ${ }^{4,23,24}$. Os resultados de estudos realizados em países desenvolvidos ${ }^{4,24}$ demonstraram que o deslocamento ativo foi um dos tipos de atividade física mais comumente praticadas pelos adolescentes, e com grande impacto sobre o tempo total de prática.

Verificou-se que pouco mais de dez por cento dos adolescentes estava simultaneamente envolvidos em diferentes tipos de prática de atividades física como, por exemplo, exercícios físicos, modalidades esportivas, deslocamentos e/ou atividades recreativas. As variações nos tipos de atividades praticadas simultaneamente ao longo da semana foram mais frequentemente observadas nos adolescentes mais jovens (14-15 anos de idade). $\mathrm{O}$ envolvimento dos mais jovens em diversos tipos de atividades físicas é importante para a manutenção ou até mesmo o aumento no nível de atividade física destes com o passar dos anos. Vivências e experiências em diferentes tipos de prática na adolescência podem gerar um conhecimento (background) sobre a participação em atividade física e tendem aumentar a possibilidade de que os jovens se mantenham fisicamente ativos também na fase adulta da vida ${ }^{25,26}$. Estudo com adolescentes australianos seguidos prospectivamente por mais de 20 anos, demonstrou que a transferência da prática de atividade física da infância para a idade adulta dependia da variedade de domínios ou tipos de atividades realizadas e o quanto antes esses adolescentes se envolviam em atividades físicas ${ }^{11}$.
Uma das principais limitações deste estudo foi ter utilizado um instrumento baseado em autorrelato dos adolescentes para mensurar a atividade física, fator que pode ter subestimado os níveis de práticas ${ }^{27}$. Porém, a análise de tipos de prática de atividade física não seria logisticamente viável por meio de medidas objetivas como, por exemplo, a do acelerômetro. Outra limitação foi ter considerado as atividades físicas que foram realizadas na semana anterior a coleta de dados. Desta forma, pode ser que as atividades referidas não representem os padrões habituais de atividade física, mas sim o padrão atual dos adolescentes. Mas este estudo tem pontos fortes que merecem ser destacados: foi realizado em amostra representativa de escolares da rede pública e privada, aspecto que aumentou a precisão das estimativas; o instrumento utilizado foi validado e a coleta de dados foi realizada por equipe treinada.

Pode-se concluir neste estudo que as atividades mais praticadas pelos adolescentes foram o deslocamento ativo e a participação em modalidades esportivas, mas, as que mais contribuíram para o escore geral de atividade física foram as práticas de esportes e de exercícios físicos. As contribuições de cada tipo de prática para o escore geral de atividade física variaram com as características sociodemográficas dos adolescentes. Destaca-se a expressiva contribuição dos esportes para quase todos os subgrupos investigados (exceto no sexo feminino), o grande dispêndio de tempo nos exercícios físicos, em especial no sexo feminino e nos que estudavam em escolas privadas, e o baixo poder explicativo do deslocamento ativo para o escore geral de atividade física.

Com base nos achados do presente estudo, recomenda-se que futuras intervenções para aumentar os níveis de atividade física direcionem ações que incentivem e deem suporte a prática de esportes (por exemplo, a criação de programas comunitários voltados a essas práticas, promoção de competições esportivas no tempo lazer), fortalecimento das aulas de educação física escolar no ensino básico (melhora na infraestrutura física dos locais de aula, seriação de conteúdos, sistematização curricular, otimização da formação profissional) e maior diversificação das modalidades esportivas ofertadas como conteúdos nessa disciplina. Em relação ao uso do deslocamento ativo, o estímulo e o incentivo por parte dos pais e/ou familiares e ações de infraestrutura física (calçadas, conectividade das ruas etc.), planejamento urbano e segurança por parte das autoridades competentes, são ações que devem 
ser realizadas para promover esse tipo de prática entre os adolescentes. Em decorrência do aumento da participação dos adolescentes em exercícios físicos é necessário o desenvolvimento de estudos para identificar onde e como estas atividades estão sendo realizadas, tendo em vista que as mesmas requerem, na maioria das vezes, a supervisão de profissionais habilitados para prescrever e supervisionar a forma de execução, o controle de intensidade e o volume para alcançar os benefícios à saúde.

\section{Colaboradores}

G Mendonça participou da concepção do artigo, análise dos dados, revisão de literatura e redação do manuscrito. LA Cheng participou da revisão de literatura e redação do manuscrito. JC Farias Júnior participou de todas as etapas do estudo, incluindo concepção do artigo, coleta e análise dos dados e revisão crítica do manuscrito.

\section{Agradecimentos}

À Coordenação de Aperfeiçoamento de Pessoal de Nível Superior (Capes), pela bolsa de estudos e pesquisa. À Secretaria de Educação da Paraíba, aos gestores, professores e alunos das escolas que participaram do estudo. Aos estagiários do Curso de Educação Física que fizeram parte da equipe de coleta de dados. 


\section{Referências}

1. Hallal P, Andersen LB, Bull FC, Guthold R, Haskel W, Ekelund U. Global physical activity levels: surveillance progress, pitfalls, and prospects. Lancet 2012; 380(9838):247-257.

2. Townsend N, Wickramasinghe K, Williams J, Bhatnagar P, Rayner M. Physical Activity Statistics 2015. London: British Heart Foundation; 2015.

3. Bacil E, Rech C, Hino A. Padrões de atividade física em escolares de Ponta Grossa, Paraná. Rev Bras Ativ Fis Saude 2013; 18(2):177-185.

4. Slingerland M, Borghouts LB, Hesselink MK. Physical activity energy expenditure in Dutch adolescents: contribution of active transport to school, physical education, and leisure time activities. J Sch Health 2012; 82(5):225-232.

5. Silva DAS, Silva RJS. Padrão de atividade física no lazer e fatores associados em estudantes de Aracaju-SE. Rev Bras Ativ Fis Saude 2008; 13(2):94-101.

6. Bauman AE, Reis RS, Sallis JF, Wells JC, Loos RJ, Martin BW. Correlates of physical activity: why are some people physically active and others not? Lancet 2012; 380(9838):258-271.

7. Azevedo Junior MR, Araújo CLP, Pereira FM. Physical and sports activities in adolescence: changes in choices over the last decades. Rev Bras Educ Fis Esp 2006; 20:5158.

8. Coll CVN, Knuth AG, Bastos JP, Hallal PC, Bertoldi AD. Time trends of physical activity among Brazilian adolescents over a 7-year period. J Adolesc Health 2014; 54(2):209-213.

9. Brazil Economic Classification Criterion. [database on the Internet] 2009 [cited November $26^{\text {th }}, 2009$ ]. Available from: http://www.abep.org/new/codigosCondutas.asx.

10. Farias Júnior JC, Lopes AS, Mota J, Santos MP, Ribeiro JC, Hallal PC. Validity and reproducibility of a physical activity questionnaire for adolescents: adapting the Self-Administered Physical Activity Checklist. Rev Bras Epidemiol 2012; 15(1):198-210

11. Cleland V, Dwyer T, Venn A. Which domains of childhood physical activity predict physical activity in adulthood? A 20-year prospective tracking study. Br J Sports Med 2012; 46(8):595-602.

12. Brasil. Ministério do Esporte (ME). Diagnóstico Nacional do Esporte - Caderno 1. Brasília: ME; 2015.

13. Silva SM, Knuth AG, Duca GFD, Camargo MBJ, Cruz $\mathrm{SH}$, Castagno V, Menezes AMB, Hallal PC. Individual and collective sports practice and associated factors among adolescents belonging to a birth cohort study. Rev Bras Educ Fis Esp 2009; 23(3):263-274.

14. Hallal PC, Victora CG, Azevedo MR, Wells JCK. Adolescent physical activity and health: a systematic review. Sports Med 2006; 36(12):1019-1030.

15. Janssen I, LeBlanc AG. Systematic review of the health benefits of physical activity and fitness in school-aged children and youth. Int J Behav Nutr Phys Act 2010; $7: 40$.
16. Barroso DR, Almeida LIR, Kulnig AM. Mídia e construção da imagem corporal em adolescentes do gênero feminino. Rev Bras Reabilitaçao e Atividade Fisica 2012; 1:53-62.

17. Mota J, Esculcas C. Leisure-time physical activity behavior: structured and unstructured choices according to sex, age, and level of physical activity. Int J Behav Med 2002; 9(2):111-121.

18. Bengoechea EG, Sabiston CM, Ahmed R, Farnoush M Exploring links to unorganized and organized physical activity during adolescence: the role of gender, socioeconomic status, weight status, and enjoyment of physical education. Res Q Exerc Sport 2010; 81(1):7-16.

19. Faulkner GE, Buliung RN, Flora PK, Fusco C. Active school transport, physical activity levels and body weight of children and youth: a systematic review. Prev Med 2009; 48:3-8.

20. Panter JR, Jones AP, van Sluijs EM. Environmental determinants of active travel in youth: A review and framework for future research. Int J Behav Nutr Phys Act 2008; 5:34.

21. Stewart O. Findings from research on active transportation to school and implications for safe routes to school programs. J Plan Lit 2011; 26(2):127-150.

22. Larouche R, Barnes J, Tremblay MS. Too far to walk or bike? Can J Public Health 2013; 104(7):e487-e489.

23. Carlson JA, Sallis JF, Kerr J, Conway TL, Cain K, Frank LD, Saelens BE. Built environment characteristics and parent active transportation are associated with active travel to school in youth age 12-15. Br J Sports Med 2014; 48(22):1634-1639.

24. Yan AF, Voorhees CC, Beck KH, Wang MQ. A Social Ecological Assessment of Physical Activity among Urban Adolescents. Am J Health Behav. 2014; 38:379-391.

25. Telama R. Tracking of physical activity from childhood to adulthood: a review. Obes Facts 2009; 2(3):187-195.

26. Telama R, Yang X, Leskinen E, Kankaanpää A, Hirvensalo M, Tammelin T, Viikari JS, Raitakari OT. Tracking of physical activity from early childhood through youth into adulthood. Med Sci Sports Exerc 2014; 46(5):955962.

27. Ekelund U, Tomkinson G, Armstrong N. What proportion of youth are physically active? Measurement issues, levels and recent time trends. Br J Sports Med 2011; 45(11):859-865.

Artigo apresentado em 16/02/2016

Aprovado em 18/08/2016

Versão final apresentada em 20/08/2016 
